\title{
CURRENT FLOW IN RECTANGULAR CONDUCTORS
}

\author{
B.y H. Fletcher Moulton.
}

[Received January 3rd, 1905.-Read January 12th, 1905.]

THE simplest case of current flow in two dimensions is that of electricity flowing across a rectangular plate the two opposite sides of which are the electrodes. I $\mathrm{am}$, however, unacquainted with any formula dealing with the cases in which the electrodes do not extend over the whole of these sides, and it is difficult to see how any approximetion based on a priori reasoning can be made for these cases.

By the use of Schwarz's transformation, however, the results come out in a very simple manner.

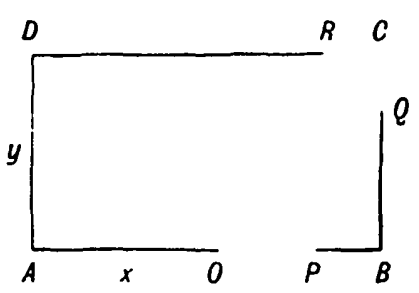

(1)

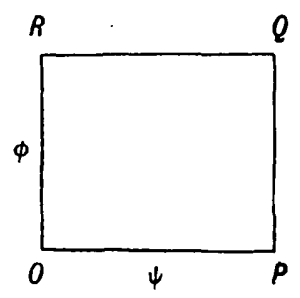

$(2)$

Let (1) be the $x, y$ diagram, the electrodes being $O P$ and $Q R$. Let (2) be the $\phi$, $\psi$ diagram. Writing, as usual, $z=x+\iota y, w=\psi+\iota \phi$, and for simplicity taking $\sigma$, the specific resistance, as unity, we put

$$
d z=\frac{1 t}{\sqrt{\{(t-u)(t-\beta)(t-\gamma)(t-\delta)\}},}
$$

$a, \beta, \gamma, \delta$ being arbitrary quantities subject to the one condition that $(\beta-\gamma)(\alpha-\delta) /(a-\gamma)(\beta-\delta)=\kappa^{2}$, where $\alpha$ is chosen so that the ratio of the periods $K^{\prime} / K=A D / A B$. Then $\kappa$ is immediately found from Legendre's tirst table. Again, put

$$
d w=\frac{d t^{\prime}}{\sqrt{ }\left\{\left(t^{\prime}-u^{\prime}\right)\left(t^{\prime}-\beta^{\prime}\right)\left(t^{\prime}-\gamma^{\prime}\right)\left(t^{\prime}-\delta^{\prime}\right)\right\}},
$$

where $\left(\beta^{\prime}-\gamma^{\prime}\right)\left(a^{\prime}-i^{\prime}\right) /\left(a^{\prime}-\gamma^{\prime}\right)\left(\beta^{\prime}-o^{\prime}\right)=l^{2}$, the modulus $l^{2}$ being such that on the $\psi, \phi$ diagram the ratio of corresponuing periods, viz., $L^{\prime} / L=O L^{\prime} / O P$, i.e., $L^{\prime} / L=$ equivalent resistance of plate. 
The quantity $l$, of course, is at present undetermined, and it is our purpose to determine it. We have

and

$$
\begin{aligned}
t & =\frac{a(\beta-\delta)-\beta(a-\delta) \mathrm{sin}^{2} m z(\bmod \alpha) .}{\beta-\delta-(a-\delta) \sin ^{2} m \cdot z(\bmod \kappa)} \\
t^{\prime} & =\frac{\alpha^{\prime}\left(\beta^{\prime}-\delta^{\prime}\right)-\beta^{\prime}\left(a^{\prime}-\delta^{\prime}\right) \operatorname{sn}^{2} m^{\prime} w(\bmod l)}{\beta^{\prime}-\delta^{\prime}-\left(\alpha^{\prime}-\delta^{\prime}\right) \sin ^{2} \cdot m^{\prime} w(\bmod l)},
\end{aligned}
$$

where $m=\frac{1}{2} \sqrt{ }\{(\alpha-\gamma)(\beta-\delta)\}, m^{\prime}=\frac{1}{2} \sqrt{ }\left\{\left(\alpha^{\prime}-\gamma^{\prime}\right)\left(\beta^{\prime}-\delta^{\prime}\right)\right\}$.

Since we have only one relation between $\alpha, \beta, \gamma$, and $\delta$, and between $\alpha^{\prime}, \beta^{\prime}, \gamma^{\prime}$, and $\delta^{\prime}$, we can take $m$ and $n \iota^{\prime}$ to be unity.

We can also simplify the problem without loss of generality by assuming the units such that $A B=K, O P=L$, and therefore $A D=K^{\prime}$, $O R=L^{\prime}$.

Now there is one and only one solution of the equation $\nabla^{2} v=0$ over the area $A B C D$ with given boundary conditions.

By the transformation adopted the boundary in the $z$ plane becomes the real axis in the $t$ plane, and the area $A B C D$ becomes the part of the $t$ plane above the real axis. So also the boundary in the 10 plane becomes the real axis in the $t^{\prime}$ plane, and the area $O P Q R$ becomes the part of the $t^{\prime}$ plane above the real axis.

If, therefore, we put $t=t^{\prime}$, and so choose the constants that each point of the real axis represents corresponding points on the $z$ and $w$ planes, we shall arrive at the required relation between $z$ and $w$.

'To do this we must determine $\alpha^{\prime}, \beta^{\prime}, \gamma^{\prime}$, and $\delta^{\prime}$, and we do so by putting $t=t^{\prime}$ at $O, P, Q$, and $R$. Let the co-ordinates of $O, P, Q$, and $R$ in the $z$ plane be $z_{1}, z_{2}, z_{3}, z_{4}$. In the $w$ plane they are $O, K, K+\iota K^{\prime}, \iota K^{\prime}$.

'Then

$$
\begin{aligned}
& \alpha^{\prime}=\frac{a(\beta-\delta)-\beta(\alpha-\delta) \mathrm{sn}^{2} z_{1}}{\beta-\delta-(a-i) \mathrm{sn}^{2} ._{1}}, \\
& \delta^{\prime}=\frac{a(\beta-\delta)-\beta(\alpha-\delta) \mathrm{sn}^{2} z_{2}}{\beta-\delta-(\alpha-\delta) \mathrm{sn}^{2} z_{2}}, \\
& \gamma^{\prime}=\frac{a(\beta-\delta)-\beta(a-\delta) \mathrm{sn}^{2} z_{3}}{(\beta-\delta)-(\alpha-\delta) \mathrm{sn}^{2} z_{3}}, \\
& \beta^{\prime}=\frac{a(\beta-\delta)-\beta(\alpha-\delta) \mathrm{sn}^{2} z_{4}}{(\beta-\delta)-(\alpha-\delta) \mathrm{sn}^{2} z_{4}} ;
\end{aligned}
$$

therefore $\quad l^{2}=\frac{\left(\beta^{\prime}-\gamma^{\prime}\right)\left(\alpha^{\prime}-\delta^{\prime}\right)}{\left(\alpha^{\prime}-\gamma^{\prime}\right)\left(\beta^{\prime}-\delta^{\prime}\right)}=\frac{\left(\mathrm{sn}^{2} z_{4}-\mathrm{sn}^{2} z_{9}\right)\left(\mathrm{sn}^{2} z_{1}-\mathrm{sn}^{2} z_{2}\right)}{\left(\operatorname{sn}^{2} z_{1}-\mathrm{sn}^{2} z_{9}\right)\left(\mathrm{sn}^{2} z_{4}-\mathrm{sn}^{2} z_{2}\right)}$.

If the electrodes both commence at the same end, $l^{2}=\mathrm{sn}^{2} z_{2} / \mathrm{sn}^{2} z_{\mathrm{y}}$. If, in addition, they are equal, then $\operatorname{sn}^{2} z_{3}=1 / \kappa^{2} \operatorname{sn}^{2} z_{2}$ and $l^{2}=\kappa^{2} \sin ^{4} z_{2}$. 
In the latter case, however, we get a simpler transformation by putting

$$
A D / A B=2 K^{\prime} / K, \quad O R / O P=2 L^{\prime} / L,
$$

and putting

$$
\operatorname{dn}(z, \kappa)=t=\operatorname{dn}(w, l) .
$$

It is at once seen that $t$ is real round the boundaries, and that, when $z=0, t=1$ and $w=0$, and, when $z=2 \iota K^{\prime}, t=-1$ and $w=2 \iota L^{\prime}$. If now $\kappa^{2} \mathrm{sn}^{2} z_{2}=l^{2}$, then $\operatorname{dn}(z, \kappa)=t=\operatorname{dn}(w, l)$ at all points.

Curves of current flow for $z_{2}=\cdot 1 \mathrm{~K}$ have been drawn from this formula in the case where $A D=2 A B$.

In the general case the formula giving the equipotential lines and the. lines of current flow is

$$
\operatorname{sn}^{2}(w, l)=\frac{\mathrm{sn}^{2} z-\mathrm{sn}^{2} z_{1}}{\mathrm{sn}^{2} z-\mathrm{sn}^{2} z_{4}} \frac{\mathrm{sn}^{2} z_{4}-\mathrm{sn}^{2} z_{2}}{\mathrm{sn}^{2} z_{1}-\mathrm{sn}^{2} z_{2}} .
$$

The calculation of the lines of current flow in the above-named case where $A D=2 A B$ and $z_{2}=2 K$ showed a very great increase of current density at the outer ends of the electrode. Theoretically the density would become infinite, but, of course, in practice the very great increase of current flow in this part of the electrode would cause a drop of potential.

The following examples of the equivalent resistances of a square conductor of unit specific resistance according to the position of the electrode may also prove interesting. They show that the increase of resistance due to the orowding tngether of the lines of current flow on their approach to a small electrode is by no means unimportant.

In each case the total length of an electrode is one-fifth of the side of the square.

(1) Electrodes opposite to each other and in the middle. Resistance $=1 \cdot 745$.

(2) Electrodes opposite to each other, but at one end. Resist. ance $=2 \cdot 408$.

(3) Electrodes on opposite sides, but at opposite corners. Resistance $=2 \cdot 589$.

(4) Electrodes bent equally round opposite corners. Resistance $=3 \cdot 027$.

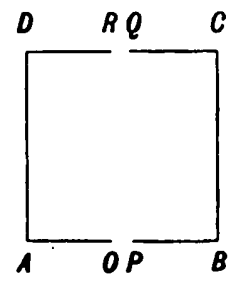

(1)

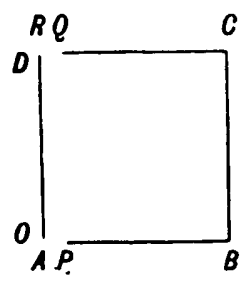

(2)

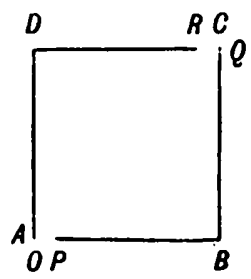

(3)

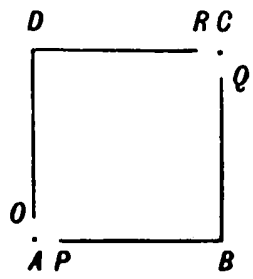

(4) 
Schwarz's transformation also entables us to find the resistance of a plane rectilinear conductor one of whose angles is a right angle, the electrodes extending over the whole of opposite sides.
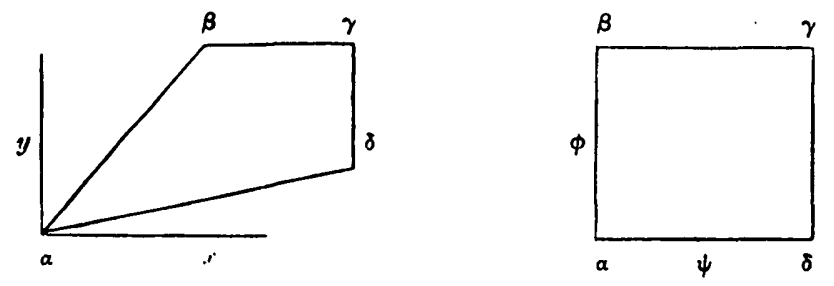

Let $a \beta y \delta$ be the conductor, $\beta \gamma \delta$ being a right angle, and construct the $x, y$ and $\psi, \phi$ diagrams as before. where

$$
\text { Let } \quad d z=\frac{c_{1} d t}{\sqrt{ }\left\{(t-\alpha)(t-\beta)(t-\gamma)(t-\partial)^{\prime}(t-\alpha)^{p}(t-\beta)^{4}(t-\delta)^{r}\right.},
$$

$$
p+\frac{1}{2}=1-\frac{\angle \delta a \beta}{\pi}, \quad q+\frac{1}{2}=1-\frac{\angle a \beta \gamma}{\pi} . \quad r+\frac{1}{2}=1-\frac{\angle a \dot{\gamma} \gamma}{\pi} .
$$

Let

$$
d w=\frac{c_{2} d t}{\sqrt{ }\{(t-a)(t-\beta)(t-\gamma)(t-\delta)\}} .
$$

Then

$$
d z=\frac{c d w}{(t-\alpha)^{p}(t-\beta)^{\prime}(t-\delta)^{r}}, \text { where } \quad c=\frac{c_{1}}{c_{2}} \text {; }
$$

but $\quad t=\frac{\alpha(\beta-\hat{o})-\beta(\alpha-\delta) \operatorname{sn}^{2} m w}{(\beta-\delta)-(u-j) \operatorname{sn}^{2} m \cdot v}\left[\bmod \sqrt{\left\{\frac{(u-\gamma)(\beta-\delta)}{(u-\delta)(\beta-\gamma)}\right)}\right]$,

where $m=\frac{1}{2} \sqrt{ }\{(\alpha-\gamma)(\beta-\delta)\}$ and can be put equal to unity without loss of generality.

We can also choose co-ordinates so that in the $\phi, \psi$ diagram ao $=K$,

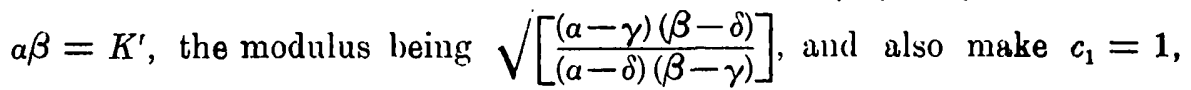
$c_{2}=1$. Then

$$
\begin{gathered}
t-a=\frac{(a-\beta)(\alpha-\delta) \operatorname{sn}^{2} w}{(\beta-\delta)-(\alpha-\delta) \operatorname{sn}^{2} w}, \quad t-\beta=\frac{(\alpha-\beta)(\beta-\delta)}{(u-\delta)-(\beta-\delta) \operatorname{sn}^{2} w}, \\
t-\delta=\frac{(\alpha-\delta)(\beta-\delta) \operatorname{cn}^{2} w}{(\alpha-\delta)-(\beta-\delta) \operatorname{sn}^{2} w}
\end{gathered}
$$

so that $\quad d z=\frac{c d w\left[(\beta-\delta)-(a-\delta) \sin ^{2} w\right]^{p+q+r}}{(a-\beta)^{p+q}(\alpha-\delta)^{p+r}(\beta-\delta)^{q+r} \operatorname{sn}^{2 p} w \mathrm{cn}^{3 r} w}$.

or, since $p+q+r=0$, we have

$$
d z=c^{\prime} d w \sin ^{-2 p} w \mathrm{cn}^{-2 r} w .
$$


If now the co-ordinates of $\beta$ and $\delta$ in the $x, y$ plane are $a+i b$ and $c+\iota d$, we get

$$
\frac{a+\iota b}{c+\iota d}=\frac{\left.\int_{0}^{K^{\prime}} d w \mathrm{sn}^{-2 p} w \mathrm{cn}^{-2 r} w\right)}{\int_{0}^{K} d w \mathrm{sn}^{-2 p} . w \mathrm{cn}^{-2 r} w}=\frac{\iota^{1-2 p} \int_{0}^{K^{\prime \prime}} d u \mathrm{sn}^{-2 p}\left(u, \kappa^{\prime}\right) \mathrm{cn}^{2(p+r)}\left(u, \kappa^{\prime}\right)}{\int_{0}^{K} d w \sin ^{-2 p} w \mathrm{cn}^{-2 r} w},
$$

which can easily be proved to be the sune as

$$
\iota^{1-2 p} \frac{\Gamma\left(\frac{1}{2}+p+r\right) \Gamma^{2}(1-p-r) F\left(\frac{1}{2}, \frac{1}{2}-p, 1+r, \kappa^{\prime 2}\right)}{\Gamma(1+r) \Gamma\left(\frac{1}{2}-r\right) F^{\prime}\left(\frac{1}{2}, \frac{1}{2}-p, 1-p-r, \kappa^{2}\right)},
$$

$F()$ denoting the hypergeometric series.

From this we can determine $\kappa^{2}$ by approximation and consequently the resistance of the conductor. This approximation can be facilitated when $\kappa$ is either very suiall or nearly unity by observing that

and

$$
F\left(\frac{1}{2}, \frac{1}{2}-p, 1-p-r, x\right)
$$

$$
l^{\prime}\left(\frac{1}{2}, \frac{1}{2}-p, 1+r, 1-x\right)
$$

are both solutions of the equation

$$
y^{\prime \prime}+\frac{1-p-r-(2-p) x}{x(1-x)} y^{\prime}-\frac{\frac{1}{2}\left(\frac{1}{2}-p\right)}{x(1-x)} y=0 .
$$

Call these two particular solutions $y_{1}$ and $y_{2}$.

Another solution in powers of $x$ is

$$
y_{3}=x^{p+r} F\left(\frac{1}{2}+p+r, \frac{1}{2}+r, 1+p+r, x\right),
$$

and another in powers of $(1-x)$ is

$$
y_{4}=(1-x)^{-r} F\left(\frac{1}{2}-p-r, \frac{1}{2}-r, 1-r, 1-x\right) .
$$

Since the above differential equation is of the second degree, we have

$$
y_{1}=A y_{2}+B y_{4}, \quad y_{2}=C y_{1}+D y_{3},
$$

where $A, B, C$, and $L$ ) are independent of $x$ and are the same for all quadrilaterals with the same angles.

In the case where $r=0$, i.e., where two adjacent angles are right angles, we find that $y_{4}$ degenerates into $y_{2}$, but in this case another solution in powers of $(1-x)$ is

$$
\begin{aligned}
y_{1}=-\log (1-x) F\left(\frac{1}{2}, \frac{1}{2}-p, 1,1-x\right) \\
+\Sigma \frac{\frac{1}{2} \cdot \frac{3}{2}\left(n-\frac{1}{2}\right)\left(\frac{1}{2}-p\right)\left(\frac{3}{2}-p\right) \ldots\left(n-\frac{1}{2}-p\right)}{(n !)^{2}} c_{n}(1-x)^{n}
\end{aligned}
$$


where

$$
c_{n}=2 \sum_{1}^{n} \frac{1}{s}-\sum_{1}^{n} \frac{1}{s-\frac{1}{2}}-\sum_{1}^{n} \frac{1}{s-\frac{1}{2}-p} .
$$

Now, since this equation is linear and of the second degree, we have

$$
y_{2}=A y_{\mathrm{r}}+B y_{3}, \quad y_{1}=C y_{2}+D y_{1},
$$

where $A, B, C, D$ are constants for a given value of $p$.

In employing this method when $\kappa$ is small, i.e., the resistance is large. we approximate rapidly by means of the equation

$$
\frac{a}{c}=\cos a \frac{\Gamma\left(\frac{1}{2}+p\right) \Gamma(1-p)}{\Gamma\left(\frac{1}{2}\right)}\left(A+B \frac{y_{3}}{y_{1}}\right) ;
$$

and, when $\kappa$ is nearly unity and the resistance is small,

$$
\frac{c}{a}=\sec a \frac{\Gamma\left(\frac{1}{2}\right)}{\Gamma\left(\frac{1}{2}+p\right) \Gamma^{\prime}(1-p)}\left(C+D \frac{y_{4}}{y_{2}}\right) .
$$

The case of $r=0$ and $p=\frac{1}{4}$ gives the resistance of a plate when the electrodes are of the form which is occasionally used, in which the current flows from one square to a square symmetrically placed round the first. It is clear from symmetry that the lines bisecting the sides at right angles and the diagonals are lines of flow. T'ie resistance therefore is $\frac{1}{8}$ of that of the quadrilateral $\alpha \beta, \delta$.

If now $\alpha \delta$ is $x$ and $\alpha \beta$ is $c+\iota$, we get

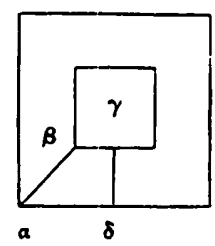

$$
\frac{c}{a}=A^{\prime}+B^{\prime} \frac{y_{3}}{y_{1}} \quad \text { or } \quad \frac{a}{c}=C^{\prime}+D^{\prime} \frac{y_{1}}{y_{2}} .
$$

The values to three places of $A^{\prime}, B^{\prime}, C^{\prime}, D^{\prime}$ are

$$
A^{\prime}=1, \quad B^{\prime}=-\cdot 457, \quad C^{\prime}=1 \cdot 605, \quad D^{\prime}=-\cdot 318 .
$$

If $k$ is small, it muy be outside the limits of Legendre's tables, but we can approximate conveniently to the resistance $K^{\prime} / K$ by means of the formula

$$
K^{\prime}=P K+Q\left\{K \log \kappa^{2}+\frac{\pi}{2} \sum_{1}^{\infty} \kappa^{21}\left(\frac{1}{2} \ldots n-\frac{1}{2}\right) \sum_{1}^{n} \frac{1}{11\left(n-\frac{1}{2}\right)}\right\}
$$

where $P=\cdot 883, Q=-\cdot 318=D^{\prime}$; and, since $K=\frac{1}{2} \pi$ nearly and $\log \kappa^{2}$ is large, we get $R=P+Q \log \kappa^{2}$. But, if $\kappa$ is very small, $B^{\prime} \kappa^{\frac{1}{3}}=c / a-1$, and to a first approximation $R=-1.28 \log \frac{a-c}{a}$. If, however, $\kappa^{\prime}$ is small, we have $\log \kappa^{\prime 2}=D^{\prime-1} a / c$ approxiniately, and therefore $1 / R=a / c$, which could, of course, have been foreseen. 
If the inner square is placed diamond-wise, then $\alpha \beta$ and $\gamma \delta$ are lines of How, and, if the co-ordinates of $\beta$ are $0, a$ and those of $\delta$ are $b, 0$,

$$
\frac{a}{l}=\frac{F\left(\frac{1}{2}, \frac{1}{2}, \frac{3}{4}, \kappa^{\prime 2}\right)}{F\left(\frac{1}{2}, \frac{1}{2}, \frac{3}{4}, \kappa^{2}\right)} \frac{\left\{\Gamma\left(\frac{3}{4}\right)\right\}^{2}}{\Gamma\left(\frac{1}{4}\right) \Gamma\left(\frac{5}{4}\right)} .
$$

Having determined $\kappa$ in any of the above cases, the

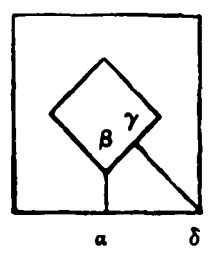
resistance is given by $K^{\prime} / K$, and the differential equations for the lines of flow and equipotential lines are

$$
d x+\iota d y=c^{\prime}(d \psi+\iota d \phi)[\operatorname{sn}(\psi+\iota \phi, \kappa)]^{-\underline{t}} .
$$

In the case of the two squares treated above the current density on the outer electrode is given by

$$
\frac{\partial \psi}{\partial x}=\operatorname{sn}^{\frac{1}{2}} \psi
$$

and on the inner by

$$
\frac{\partial \psi}{\partial x}=1 / \kappa^{\frac{1}{3}} \operatorname{sn}^{\frac{1}{3}} \psi \text {. }
$$

